\section{A novel in vitro culture system for the generation of controlled hydrodinamic stimuli}

Elia Pederzani, ${ }^{1}$ Lorenzo P. Coppadoro, ${ }^{1}$ Chiara Foglieni, ${ }^{2}$ Maria Lombardi, ${ }^{2}$ Monica Soncini, ${ }^{1}$ Gianfranco B. Fiore ${ }^{1}$

1Dipartimento di Elettronica, Informazione e Bioingegneria, Politecnico di Milano; ${ }^{2}$ Cardiovascular Research Area, IRCCS San Raffaele Scientific Institute, Milano, Italy

\section{Abstract \\ A novel device to analyze the correla- tion between hydrodinamic stimuli and atherosclerosis advancement.}

\section{Introduction}

Atherosclerosis is a chronic-degenerative pathological phenomenon that affects the arterial system, in particular the subendothelium region where the formation and progressive growth of a plaque of lipid nature, called atheroma, compromises the vascular wall homeostasis. ${ }^{1}$ In the most advanced stages of the pathology, the plaque reaches such dimensions as to partially occlude the vessel, thus causing a stenosis. In addition, plaque is susceptible to rupture that causes the release of dangerous agglomerates into the bloodstream that can cause thrombi.

Besides the systemic risk factors (age, gender, diet, other pathologies) the local hemodynamic seems to have a crucial role in the illness development. In fact, the cells are extremely sensitive to tangential stresses occurring in the vessel wall (wall shear stress, WSS), which are correlated with local fluid dynamics. It is known that the arterial districts, where atherosclerotic plaques are most frequently found, are those with disturbed fluid dynamics. Indeed, the alteration of WSS is considered the trigger of endothelium's permeabilization and consequent lipids' diffusion within the intima tunic. ${ }^{2}$

In this scenario, we have developed a novel device that allows to host human vascular tissue samples of not negligible thickness, and to apply multidirectional, complex and variable over time WSS patterns. This in vitro system represents a useful model for investigating the complex phenomena that arise in the sub-endothelium subsequent specific WSS patterns; moreover, it turns out to be a valid alternative to animal models, which today represent the only option to study the correlation between atherosclerosis and complex hydrodynamic stimuli.

\section{Materials and Methods}

The bioreactor (Figure 1) working principle is based on the combination of an input flow rate and sample rotation: thanks to this is possible to generate complex multidirectional stresses by instantly varying both the modulus and the direction of the WSS vector. The designed bioreactor consists of two main elements: the vascular sample housing (composed by a cartridge, a piston and two ferrules), to be inserted in the parallel plates flow chamber. In order to minimize the risk of tissue damage during sample handling and sample mounting within the bioreactor, we have also devised specific procedures and realized auxiliary
Correspondence: Elia Pederzani, Dipartimento di Elettronica, Informazione e Bioingegneria, Politecnico di Milano, Milano, Italy.

E-mail: elia.pederzani@polimi.it

Key words: In vitro culture system; wall shear stress; atherosclerosis.

Disclosures: The authors declare no conflict of interests.

Conference presentation: This paper was presented at the Third Centro 3R Annual Meeting - L'era delle 3R: modelli in silico, in vitro e in vivo per promuovere la ricerca traslazionale 30 September - 1 October 2021, Evento online organizzato dal Politecnico di Torino.

Received for publication: 9 July 2021.

Accepted for publication: 7 September 2021.

This work is licensed under a Creative Commons Attribution NonCommercial 4.0 License (CC BY-NC 4.0).

(C) Copyright: the Author(s), 2021

Licensee PAGEPress, Italy

Biomedical Science and Engineering 2021; 4(s1):187 doi:10.4081/bse.2021.187

accessories. The correct alignment of the endothelium with the flow channel bottom is essential to stimulate the sample with the desired WSS. This is made possible by the joint use of a proximity sensor and the threated piston adjusting the sample position. Through two gears, it is possible to transfer the rotary motion of a stepper motor to the entire sample housing module and consequently to the hosted biological sample. The device control is managed by a custom electronic interface: it actuates the motor and the pumps, and it allows the control of the circuit parameters thanks to the

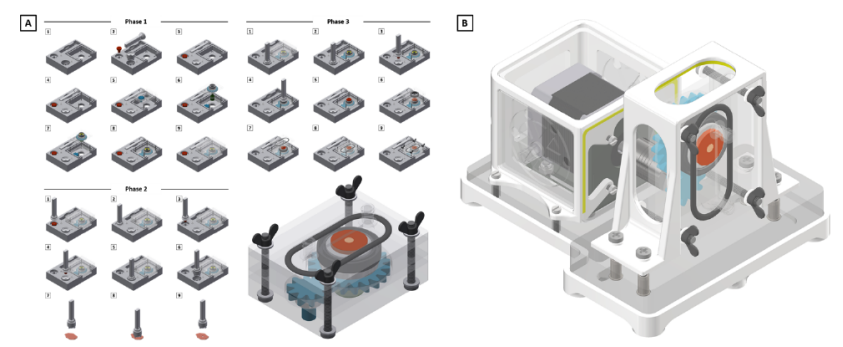

Figure 1. A) Bioreactor assembly procedure: preliminary phase, sample cutting, device closure; B) Multidirectional shear stress bioreactor: device, stepper motor, motor case.

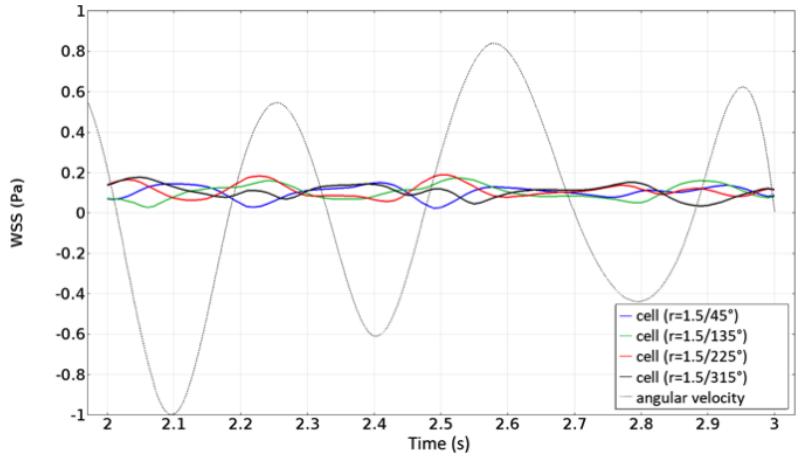

Figure 2. Physio-pathologic protocol simulation. 
connection with sensors. The protocols developed to ensure multidirectional stimulation are classified into:

i) parametric protocols, which reproduce a specific motion law associated with an OSI value chosen by the operator within the full OSI range; ii) physio-pathologic protocols, which are obtained from literature 4D-flow analyses providing a precise time course of the WSS vector in modulus and direction. ${ }^{3}$

With the intention of verify the working hypotheses, in particular the sample's stress accuracy degree acting in spite of the viscous drag forces due to the housing rotation, it is necessary to simulate each motion law, analysing if they excessively affect the fluid dynamics inside the flow channel. The fluid dynamic simulations are made by COMSOL Multiphysics ${ }^{\circledR}$ a finite element simulation software; we analysed the WSS acting on four different points, each representative of a different sample's sector for both the parametric and protocol and the physiopathologic one.

\section{Results}

The device has been successfully tested by performing preliminary bench tests. We found that the hydraulic seal is guaranteed up to $780 \mathrm{mmHg}$ and it has been verified in experiments running up to 5 days. The detection system average sensitivity has been evaluated approximately $300 \mathrm{mV} / \mathrm{mm}$. Through fluid dynamic simulations, we have verified that the sample rotation generates an acceptable deviation from the WSS target value. Figure 2 shows a physiopathologic protocol; with a constant WSS target value of $0.1 \mathrm{~Pa}$, we have observed a mean deviation of about $5-10 \%$.

\section{Discussion and Conclusions}

The analyses carried out until now have demonstrated the system efficacy of the system in generating controlled hydrodynamic stimuli; we are performing biological tests to evaluate the correlation between different stress patterns and the cellular response. The in vitro culture system represents an innovative device with respect to the current state-of-the-art enabling the possibility to investigate different stress patterns effects, no longer only on a cellular layer, but also on whole vascular samples. In perspective we will exploit this device to examine the complex mechanobiological interactions that occur in the sub-endothelial tissue in the early stage of the atherosclerosis pathology evolution.

\section{References}

1. Cunningham KS, Gotlieb AI. The role of shear stress in the pathogenesis of atherosclerosis. Lab Invest 2005;85:923.

2. Gimbrone Jr MA, García-Cardeña G. Vascular endothelium, hemodynamics, and the pathobiology of atherosclerosis. Cardiovasc Pathol 2013;22:9-15.

3. Cao K, Atkins SK, McNally A, et al. Simulations of morphotype-dependent hemodynamics in non-dilated bicuspid aortic valve aortas. J Biomech 2017;50:63-70. 